\title{
Short-term Changes on Follow-up Chest X-rays of Familial Cluster of COVID-19 Cases
}

\author{
Chun-Xue $\mathrm{Wu}^{1,2}$, Dong-Dong Rong ${ }^{1,2}$, Yan-Hong $\mathrm{An}^{1,2}$, Yi Shan ${ }^{1,2}$ and Jie $\mathrm{Lu}^{1,2}$ \\ ${ }^{1}$ Department of Radiology and Nuclear Medicine, Xuanwu Hospital, Capital Medical University, Beijing, China \\ ${ }^{2}$ Beijing Key Laboratory of Magnetic Resonance Imaging and Brain Informatics, Beijing, China
}

\begin{abstract}
In this study, we report a familial cluster of cases which included five patients and two close contacts who were confirmed to have coronavirus disease 2019 (COVID-19). These participants had received real-time reverse transcription-polymerase chain reaction (RTPCR) and chest X-rays (CXRs) before diagnosis. The follow-up CXRs of three patients in the family showed significant progression, with COVID-19 pneumonia, clinically worsening in a short period of time. Therefore, the results of follow-up CXRs in the short-term may be an adjunctive diagnostic method for COVID-19 disease diagnosis and its progression.
\end{abstract}

Key Words: Chest X-ray, COVID-19, RT-PCR, Familial clustering.

How to cite this article: Wu CX, Rong DD, An YH, Shan Y, Lu J. Short-term Changes on Follow-up Chest X-rays of Familial Cluster of COVID-19 Cases. J Coll Physicians Surg Pak 2021; 31(JCPSPCR):CR19-CR22.

\section{INTRODUCTION}

During the current pandemic of coronavirus disease 2019 (COVID-19), chest X-rays (CXRs) and not computed tomography (CT), have been employed as a first-line triage tool in many areas. This is because CXRs reduced the cross-infection risk of COVID-19. ${ }^{1-3}$ The manifestations of COVID-19 on CXRs have been described in many articles; ${ }^{4,5}$ but, to our knowledge, the experience of the value of CXR in assessing disease progression and as an ancillary diagnostic tool in COVID-19 patients has rarely been reported.

We, herein, report a familial cluster of cases which included five patients and two close contacts in January 2020 in Beijing Xuanwu Hospital, China.

All the baseline and follow-up CXRs in our hospital were completed in isolation wards. The follow-up CXRs of three patients in the family showed significant progression, with COVID-19 pneumonia clinically worsening in a short period of time.

\section{CASE REPORT}

The index patient came from Wuhan. She was of 65 years and travelled with her family (patients 3 and 4 and close contact 1 ) to visit her aunt (patient 1) in Beijing on 12 January, 2020 (Figure 1). The timeline of exposure is shown in Figure 2.

Correspondence to: Dr. Jie Lu, Department of Radiology, Xuanwu Hospital, 45 Changchun Street, Xicheng District, Beijing, China

E-mail: lujie1057@163.com

Received: October 22, 2020; Revised: December 01, 2020;

Accepted: December 21, 2020

DOI: https://doi.org/10.29271/jcpsp.2021.JCPSPCR.CR19

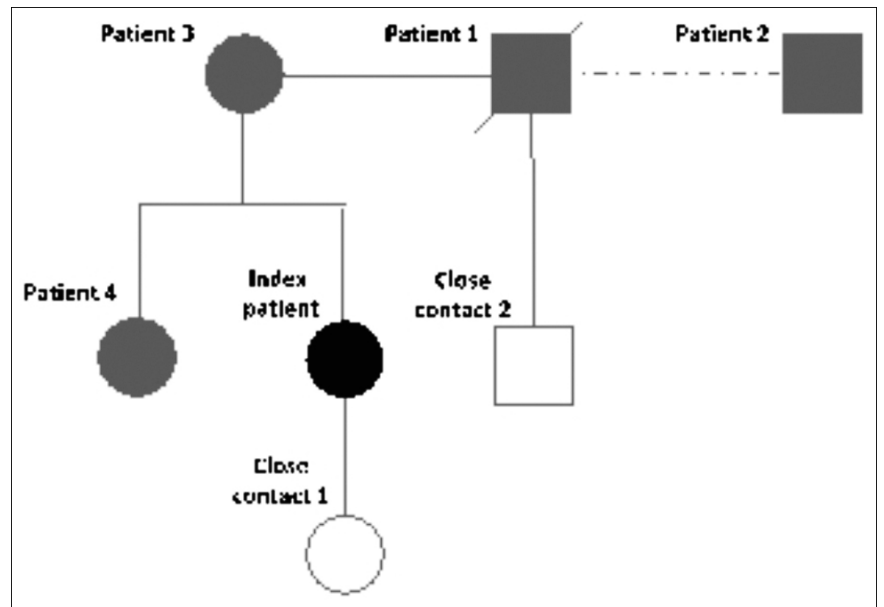

Figure 1: Patients with COVID-19 infection and close contacts in the familial clustercase.

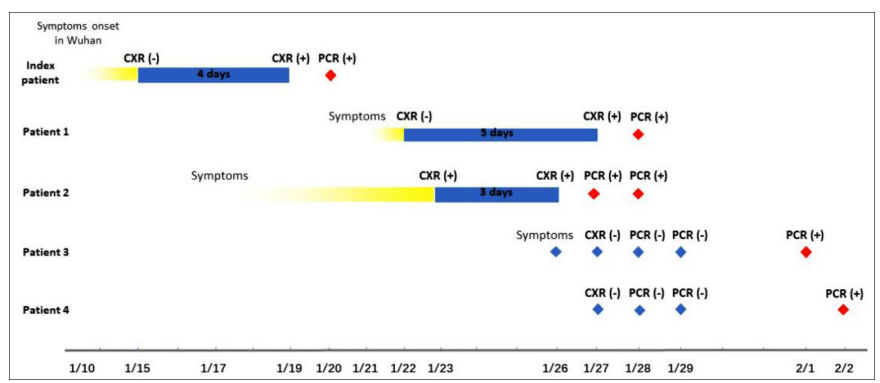

Figure 2: Timeline of exposure to index patient.

A few days before leaving Wuhan, the index patient had a cough but no fever. She had a CXR in a hospital in Beijing on 15 January because of patchy ground-glass opacity (GGO). She was given antibiotics and went back to her aunt's home. Her symptoms did not resolve and she began to wheeze. Her follow-up CXR on 19 January showed patchy consolidation (right middle lung field). 
Table I: Summary of laboratory examination results of the familial cluster infected with COVID-19.

\begin{tabular}{|c|c|c|c|c|c|}
\hline & Reference range & Patient 1 & Patient 2 & Patient 3 & Patient 4 \\
\hline C-reactive protein, mg/L & $0-10$ & 8 & 11 & 17 & 19 \\
\hline Eosinophils, $\times 109 / L$ & $0-0.3$ & 0.01 & 0.00 & 0.01 & 0.00 \\
\hline Eosinophil ratios, \% & $0.5-5$ & 0.2 & 0.00 & 0.2 & 0.0 \\
\hline Lymphocytes, ×109/L & $1-3.3$ & 0.95 & 0.91 & 1.17 & 1.01 \\
\hline Lymphocyte ratios, \% & $20-40$ & 20.0 & 31.9 & 26.2 & 24.6 \\
\hline Neutrophils, ×109/L & $1.8-6.4$ & 3.19 & 1.70 & 2.89 & 2.48 \\
\hline Neutrophil ratios, \% & $50-75$ & 67.2 & 59.7 & 64.7 & 60.6 \\
\hline White blood cell count, $\times 109 / \mathrm{L}$ & $4-10$ & 4.74 & 2.85 & 4.46 & 4.1 \\
\hline
\end{tabular}
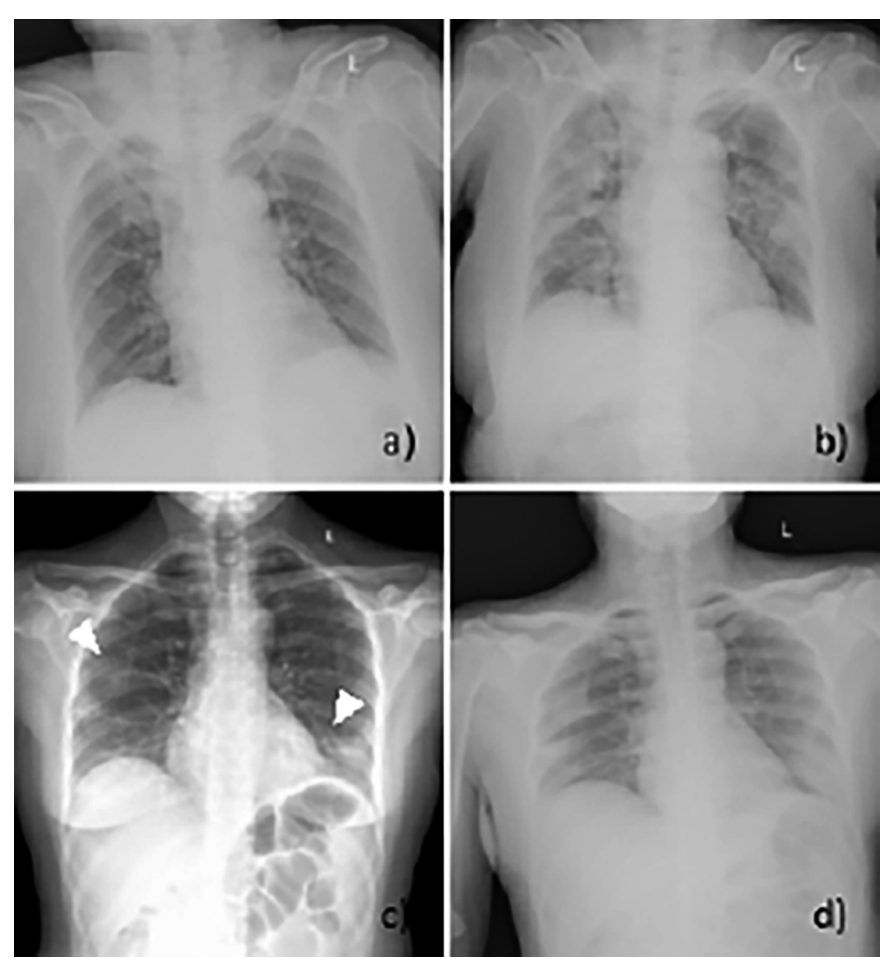

Figure 3: Manifestations of initial CXRs and follow-up CXRs of patient 1 (a, b) and patient 2 (c, d). a. Anteroposterior CXR showed no significant abnormality in the first visit of patient 1 (score $=0$ ). b. The follow-up CXR five days later showed multiple patchy consolidative lesions in both lungs, and some lesions were confluent (score = 17). c, d. Initial CXR of patient 2 showed patchy GGO in the peripheral zone of both lungs (arrowheads) (score $=4$ ). The follow-up CXR after 3 days showed that the lesions progressed with consolidation (score =7).

We used radiographic assessment of lung edema (RALE) score to assess the extent and density of infection. When the final score of the follow-up X-ray was higher than that of baseline X-ray, it was considered to be progression of disease. For index patient, her baseline RALE score was 2, and the follow-up score was 4 . That means the infection progressed in four days. The RT-PCR testing was positive on 20 January, 2020.

Patient 1, another 86-year female was admitted to our hospital because of fever and cough. The C-reactive protein level and lymphocyte count were normal and are listed in Table I. The CXR showed no significant abnormalities (Figure 3a), RALE score was 0 , so the diagnosis of COVID-19 pneumonia was not considered at that time.

Due to her exposure history, she was isolated and observed at home, and the symptoms of drowsiness and anorexia gradually appeared. The follow-up CXR showed multiple patchy GGOs in both lungs (Figure 3b) on 27 January, 2020 and the RALE score was 17 . She was diagnosed as COVID-19 infection by a positive RT-PCR test on 28 January, 2020. She died from pneumonia a week later.

Patient 2, a 47-year woman, was a housekeeper for patient 1 's family. She had a sore throat and was admitted to our hospital on 23 January, 2020. CXR showed scattered patchy GGOs in the peripheral zone of both lungs (Figure $3 c$ ) and RALE score was 4 . Because she denied contact with any other people from Wuhan, she was not diagnosed with COVID-19 pneumonia and returned home. On 26 January, she was admitted to our hospital again because of diarrhoea. The previous lesions were enlarged and showed consolidation on her follow-up CXR (Figure $3 \mathrm{~d}$ ) and the RALE score was 7. Two throat swab samples were found to be positive for COVID-19 on RT-PCR on 27 and 28 January, 2020.

Because the three patients had been confirmed as infected with COVID-19, the other members in the family (patient 3 and 4 , close contacts 1 and 2) were admitted to our hospital together on 27 January, 2020. Patient 3 (mother of the index patient) had been feverish and coughing since 26 January, while the others had no symptoms. They all had negative CXRs on admission and negative PCR tests on 28 and 29 January. During home-isolation, patient 3 and her son (patient 4) became COVID-19 positive by RT-PCR tests on 1 and 2 February, respectively. Close contact 1 (grandson of the index patient) and close contact 2 (daughter of patient 1) remained asymptomatic.

All these patients, admitted to our hospital, had normal C-reactive protein levels and lymphocyte counts at their first visits Table I. 


\section{DISCUSSION}

The results from this familial cluster case suggest that the follow-up CXRs were useful in assessing significant progression of COVID-19 pneumonia, which worsened clinically during a short period of time. The results of the follow-up CXRs also helped confirm the diagnoses of COVID-19. RT-PCR tests were all positive when the patients had abnormalities on follow-up CXRs.

In this case, initial CXRs were negative in two out of the five patients, which is a lower rate than that reported in a previous study. ${ }^{4}$ Many studies have found that CXR is insensitive in early COVID-19 infection, and that early CXRs have little value in diagnosis. ${ }^{4}$ However, in some areas, if patients stayed at home until exacerbation, initial CXRs were often abnormal on admission. In this case, all follow-up CXRs showed abnormalities five to six days after symptom onset, and the lesions progressed in three to five days after the initial CXR, which is a shorter time than that mentioned in another study. ${ }^{4}$ In our case, the index patient and patients 1 and 2 were not considered to be infected with COVID-19 at their first visits, but the diagnosis was changed after a short period of significant progress in the chest radiographs. Our case suggests that short-term follow-up CXRs could be helpful for diagnosis or alternative diagnosis when patients with mild symptoms show clinical progression.

Consolidation and GGOs were the main features of COVID-19 pneumonia on CXRs in these cases, which are consistent with the published case series. ${ }^{4,6-7}$ RT-PCR tests were all positive when patients had abnormalities on CXR in our case.

One previous study reported a patient who had significant exposure history of COVID-19 and a positive CT, but her PCR test on the throat swab was negative. ${ }^{8}$ Other articles have studied the time course of CT changes during recovery phases of COVID-19 pneumonia. ${ }^{9-10}$ Only one study reported the value of CXR and PCR for COVID-19 diagnosis and found that sensitivity of the initial CXR was still slightly lower than that of RT-PCR on throat swab. ${ }^{4}$ As there was no initial PCR performed in patients who had follow-up CXRs, we could only find that the RT-PCR was positive when the follow-up CXR was abnormal.

In conclusion, we encountered a familial cluster case, in which three members had abnormal follow-up CXRs after COVID-19 infections were confirmed by RT-PCR. The findings on follow-up CXRs showed progression when their mild symptoms worsened. We suggest that when PCR test is difficult to achieve quickly, inclusion of short-term CXR progression in the diagnostic criteria may improve the diagnosis of COVID-19 disease.

\section{FUNDING:}

Beijing Municipal Administration of Hospitals' Ascent Plan, Code: DFL20180802. The funder is responsible for overall project promotion.
PATIENTS' CONSENT:

All patients were consented to participate.

\section{CONFLICT OF INTEREST:}

The authors declared no conflict of interest.

\section{AUTHORS' CONTRIBUTION:}

CW: Planned and wrote the case report.

DR, YA: Collected the data from patients and provided the biochemical and radiological evaluation of the patients.

YS: Supervised data entry and reviewed the manuscript.

JL: Reviewed and revised the manuscript.

Every author contributed and approved the submitted version of the manuscript. Special thanks should go to my colleague Dr. Li Wei Hua, who has put considerable time into proofreading of the draft.

\section{REFERENCES}

1. Rubin GD, Ryerson CJ, Haramati LB, Sverzellati N, Kanne JP, Raoof $S$, et al. The role of chest imaging in patient management during the COVID-19 pandemic: A multi-national consensus statement from the fleischner society. Radiology 2020; 7:201365. doi: 10.1148/radiol. 2020201365.

2. Lessons from the frontline of the covid-19 outbreak - The BMJ. http://blogs.bmj.com/bmj/2020/03/20/lessons-fromthe-frontline-of-the-covid-19-outbreak/?utm_ campaign= shareaholic\&utm_medium=twitter\&utm_source=socialne twork. Accessed March 22, 2020.

3. ACR recommendations for the use of chest radiography and computed tomography (CT) for suspected COVID-19 infection | American College of Radiology. http://www. acr.org/Advocacy-and-Economics/ACR-Position-Statements/Recommendations-for-Chest-Radiography-and-CT-for-Suspected-COVID19-Infection. Accessed March 22, 2020.

4. Wong HYF, Lam HYS, Fong AH, Leung ST, Chin TWY, Lo CSY, et al. Frequency and distribution of chest radiographic findings in COVID-19 positive patients. Radiology 2019; 27:201160. doi: 10.1148/radiol.2020201160.

5. Imaging the coronavirus disease COVID-19. http: healthcare-in-europe.com/en/news/imaging-the-coronavirus-disease-covid-19.html. Accessed March 23, 2020.

6. Franquet $\mathrm{T}$, Jeong $\mathrm{YJ}$, Lam HYS, Wong HYF, Chang Y-C, Chung $M J$, et al. Imaging findings in coronavirus infections: SARS-COV, MERS-COV, and SARS-COV-2. Br J Radiol 2020; 25:20200515. doi: 10.1259/bjr.20200515.

7. Yoon SH, Lee KH, Kim JY, Lee YK, Ko H, Kim KH, et al. Chest radiographic and $\mathrm{CT}$ findings of the 2019 novel coronavirus disease (COVID-19): Analysis of nine patients treated in Korea. Korean J Radiol 2020; 21(PG-10.3348/kjr.2020. 0132):10.3348/kjr.2020.0132. doi:10.3348/kjr.2020.0132

8. Hase R, Kurita T, Muranaka E, Sasazawa H, Mito H, Yano Y. A case of imported COVID-19 diagnosed by PCR-positive lower respiratory specimen but with PCR-negative throat swabs. Infect Dis (Lond). 2020; 52(6):423-6. doi: 10.1080/23744235. 2020. 1744711.

9. Pan F, Ye TH, Sun P, Gui S, Liang B, Li LL, et al. Time course of lung changes on chest ct during recovery from 2019 
novel coronavirus (COVID-19) pneumonia. Radiology 2020; 200370. doi:10.1148/radiol.2020200370.

10. Wang YH, Dong CJ, Hu Y, Li CG, Ren QQ, Zhang X, et al.
Temporal changes of CT findings in 90 patients with COVID-19 pneumonia: A longitudinal study. Radiology 2020; 200843. doi:10.1148/radiol.2020200843. 